\title{
Multirate Numerical Integration for Stiff ODEs
}

\author{
V. Savcenco and R.M.M. Mattheij \\ Technische Universiteit Eindhoven, P.O. Box 513, 5600 MB Eindhoven, The \\ Netherlands
}

Summary. This paper contains an overview of a self-adjusting multirate method. A simple extension which allows the improvement of the efficiency of the method is introduced. The performance of the extended and the original method is compared for a test problem.

\section{Introduction}

For the numerical solution of systems of ODEs there are many methods available. These methods use time steps that are varying in time, but are constant over the components. However, there are many problems of practical interest, where the temporal variations have different time scales for different sets of the components. For example, cellular phones consist of coupled digital and analogue sub-circuits, which operate in nano- and micro-seconds, respectively. The motion of the particles around a star, which attracts mass from a secondary star, in astrophysics is described by a large system of ordinary differential equations. In this system the components, that correspond to the particles near the center, are much faster than those corresponding to the distant ones. To exploit these local time scale variations, one needs multirate methods that use different, local time steps over the components. In these methods big time steps are used for the slow components and small time steps are used for the fast ones.

Various multirate methods were developed for solving systems with different time scales. The first descriptions of multirate schemes were given by Gear and Wells [4] for multistep methods. Sand and Skelboe [9] studied the stability of backward Euler multirate methods. Multirate methods for non-stiff problems have been examined by Engstler and Lubich [3]. A multirate scheme based on the partitioned Runge-Kutta methods was introduced by Günther, Kværn $\varnothing$ and Rentrop [5]. In [1, 13, 14] multirate methods have been applied to the modeling of electrical networks. Multirate methods for hyperbolic conservation laws were studied by Constantinescu and Sandu [2]. 
A multirate method based on the Rosenbrock methods, together with a self-adjusting partitioning strategy was introduced and analyzed in $[7,10,11$, 12]. In this paper we present an overview of this method and suggest a way to improve it. The comparison of the numerical results obtained with the original and extended strategy is presented.

The paper is organized as follows. In Section 2 we will introduce the Rosenbrock methods which will be used as our basic numerical integration methods and describe the multirate time stepping strategy. The performance of the extended version of the multirate strategy for a test problem is discussed in Section 3. Finally, Section 4 contains the conclusions.

\section{A Multirate time stepping strategy}

We will consider multirate methods for solving systems of ODEs

$$
w^{\prime}(t)=F(t, w(t)), \quad w(0)=w_{0},
$$

with given initial solution $w_{0} \in \mathbb{R}^{m}$. The approximations at the global time levels $t_{n}$ will be denoted by $w_{n}$.

Our multirate time stepping strategy is based on local temporal error estimation. For a given time step $\Delta t_{n}=t_{n}-t_{n-1}$, we compute a first, tentative approximation at the new time level for all components. For those components for which the error estimator indicates that the local temporal error is larger than a given tolerance Tol, the computation is redone with smaller steps. The refinement is recursively continued until the error estimator is below Tol for all components. Schematically, with components horizontally and time vertically, the multirate time stepping is displayed in Figure 1.

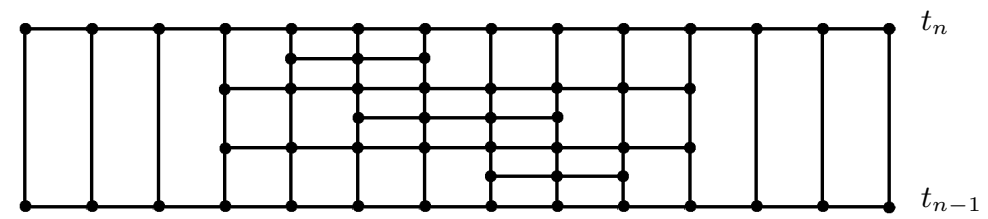

Fig. 1. Multirate time stepping for a time slab $\left[t_{n-1}, t_{n}\right]$.

In the original strategy [12], the refinement is performed by recalculating the required components with halved steps. For many problems, the time steps needed for the active components are much smaller than those needed for the slow ones. In such cases it is more efficient to immediately recompute the active components with more than two smaller steps instead of doing several halving recursive refinements. Therefore in this paper we will extend the original strategy and will assume that the number of smaller time steps at the refinement stage can be also larger than two. Using ideas from [12], it is possible to design an adaptive procedure of choosing the size of the time slabs for the extended strategy. 


\subsection{Main time integration methods}

In this paper we will use the Rosenbrock methods [6] as our basic numerical integration methods. To proceed from $t_{n-1}$ to $t_{n}=t_{n-1}+\tau$, an $s$-stage Rosenbrock method calculates

$$
\begin{gathered}
w_{n}=w_{n-1}+\sum_{i=1}^{s} b_{i} k_{i}, \\
k_{i}=\tau F\left(t_{n-1}+\alpha_{i} \tau, w_{n-1}+\sum_{j=1}^{i-1} \alpha_{i j} k_{j}\right)+\tau \frac{\partial F}{\partial w}\left(t_{n-1}, w_{n-1}\right) \sum_{j=1}^{i} \gamma_{i j} k_{j} \\
+\gamma_{i} \tau^{2} \frac{\partial F}{\partial t}\left(t_{n-1}, w_{n-1}\right), \quad i=1, \ldots, s,
\end{gathered}
$$

where $\alpha_{i j}, \gamma_{i j}, \alpha_{i}, \gamma_{i}, b_{i}$ are real parameters defining the method and $\tau$ denotes the step size. For the local error estimation withing the variable step size control we use the embedded formula

$$
\bar{w}_{n}=w_{n-1}+\sum_{i=1}^{s} \bar{b}_{i} k_{i}
$$

which uses the same $k_{i}$-values as (2), but has different weights.

\subsection{Interface treatment}

During the refinement stage, values at the intermediate time levels of components which are not refined might be needed. These values can be obtained by use of dense output built in the time integration method

$$
w_{I}\left(t_{n-1}+\theta \tau\right)=w_{n-1}+\sum_{i=1}^{s} b_{i}(\theta) k_{i}, \quad 0 \leq \theta \leq 1 .
$$

Proper interface treatment is very important for multirate schemes. Use of dense output of order lower than the order of the main time integration method can lead to order reduction.

It is well known that use of Rosenbrock methods for problems with stiff source terms can lead to order reduction. During the refinement step, sub problems with stiff source terms have to be solved. An easy applicable technique, to avoid the order reduction, was proposed in [11]. Assuming that $g(t)$ is a component of $F(t, w(t))$, this technique suggests that the source terms $g\left(t_{n-1}+\alpha_{i} \tau\right)+\gamma_{i} \tau g^{\prime}\left(t_{n-1}\right)$ in a Rosenbrock method (3) of order $p$ shall be replaced by $g_{n-1, i}$ with $\boldsymbol{g}_{n-1}=\left[g_{n-1, i}\right]$ chosen as

$$
\boldsymbol{g}_{n-1}=\sum_{k=0}^{p} \boldsymbol{B}^{k} \boldsymbol{e} \tau^{k} g^{(k)}\left(t_{n-1}\right),
$$

where $\boldsymbol{B}=\left[\alpha_{i j}+\gamma_{i j}\right] \in \mathbb{R}^{s \times s}$ and $\boldsymbol{e}=[1] \in \mathbb{R}^{s}$. 


\section{Numerical test}

In this section we will present numerical results for a test problem. We consider the behavior of both strategies: original [12] and extended (where refinement with more than two steps is possible). The results are compared to the single-rate approach, also using the same basic time integration method. As a measure for the amount of work we consider the total number of components at which solutions are computed over the complete time integration interval, multiplied by the number of stages of the method. The fact that with the multirate approach some solution components are computed several times at certain time levels is taken into account. As the basic time integration method, for solving this problem, we use the two-stage second-order Rosenbrock ROS2 method [8].

\subsection{An Inverter Chain Problem}

An inverter is an electrical sub-circuit which transforms a logical input signal to its negation. The inverter chain is a concatenation of several inverters, where the output of an inverter serves as input for the succeeding one. An inverter chain with an even number of inverters will delay a given input signal and will also provide some smoothing of the signal.

The model for $m$ inverters consists of the equations

$$
\left\{\begin{array}{l}
w_{1}^{\prime}(t)=U_{\mathrm{op}}-w_{1}(t)-\Upsilon g\left(u_{\mathrm{in}}(t), w_{1}(t)\right), \\
w_{j}^{\prime}(t)=U_{\mathrm{op}}-w_{j}(t)-\Upsilon g\left(w_{j-1}(t), w_{j}(t)\right), \quad j=2, \ldots, m,
\end{array}\right.
$$

where

$$
g(u, v)=\left(\max \left(u-U_{\text {thres }}, 0\right)\right)^{2}-\left(\max \left(u-v-U_{\text {thres }}, 0\right)\right)^{2} .
$$

The coefficient $\Upsilon$ serves as stiffness parameter. We solve the problem for a chain of $m=500$ inverters with $\Upsilon=100, U_{\text {thres }}=1$ and $U_{\text {op }}=5$. The initial condition is

$$
w_{j}(0)=6.247 \cdot 10^{-3} \text { for } j \text { even, } \quad w_{j}(0)=5 \text { for } j \text { odd } .
$$

The input signal is given by

$$
u_{\text {in }}(t)= \begin{cases}t-5 & \text { for } 5 \leq t \leq 10 \\ 5 & \text { for } 10 \leq t \leq 15 \\ \frac{5}{2}(17-t) & \text { for } 15 \leq t \leq 17 \\ 0 & \text { otherwise }\end{cases}
$$

An illustration of the solution for some of the even components is given in Figure 2. 


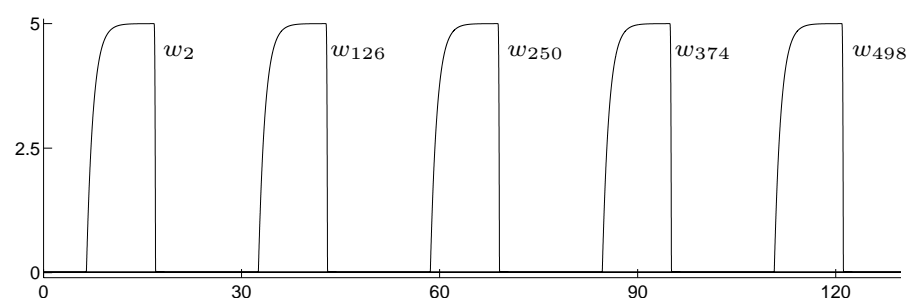

Fig. 2. Solution components $w_{j}(t), j=2,126,250,374,498$, for problem (7)-(10).

In Table 1 the errors at output time $T=130$ (measured in the maximum norm with respect to an accurate reference solution) together with the amount of work are presented for several tolerances for the single-rate method and the extended multirate strategy. The speedup for both original and extended strategies is calculated.

It is seen from the table that a substantial improvement in amount of work is obtained for this problem. For the single-rate scheme, the amount of work is almost 18 times larger than for the extended multirate scheme. Moreover, the error behavior of the multirate scheme is very good. We can also see that for this problem we get a considerably larger speedup for the extended strategy compared to the original strategy.

Table 1. Errors and work amount for problem (7)-(9).

\begin{tabular}{|r||c|c||c|c||r|r|}
\hline \multicolumn{1}{|c||}{} & \multicolumn{2}{c||}{ Single-rate } & \multicolumn{2}{c||}{ Multirate (extended) } & \multicolumn{2}{c|}{ Speedup } \\
\hline tol & error & work & error & \multicolumn{1}{c|}{ work } & original & extended \\
\hline \hline $5 \cdot 10^{-4}$ & $1.44 \cdot 10^{-1}$ & 45649872 & $1.47 \cdot 10^{-2}$ & 2846068 & 8.7 & 16.0 \\
\hline $10^{-4}$ & $3.94 \cdot 10^{-2}$ & 94524592 & $7.16 \cdot 10^{-3}$ & 5512400 & 13.0 & 17.1 \\
\hline $5 \cdot 10^{-5}$ & $1.37 \cdot 10^{-2}$ & 131413560 & $3.24 \cdot 10^{-3}$ & 6980676 & 13.5 & 18.8 \\
\hline $10^{-5}$ & $2.04 \cdot 10^{-3}$ & 287207252 & $9.22 \cdot 10^{-4}$ & 14332486 & 11.1 & 20.0 \\
\hline
\end{tabular}

\section{Conclusions}

In this paper we made on overview and extended the multirate time stepping strategy introduced in $[7,10,11,12]$.

As seen from the numerical tests, the efficiency of time integration methods can be significantly improved by using large time steps for inactive components, without sacrificing accuracy. Comparing the results obtained for the original and the extended strategies, we do have preference for the extended 
approach, where the values of the active components can be recalculated by the use of more than two smaller time steps.

\section{References}

1. A. Bartel, M. Günther, A multirate W-method for electrical networks in state space formulation. Journal Comp. Appl. Math., 147, 411-425 (2002)

2. E.M. Constantinescu, A. Sandu, Multirate time stepping methods for hyperbolic conservation laws. Journal on Scientific Computing, 33, 239-278 (2007)

3. C. Engstler, C. Lubich, Multirate extrapolation methods for differential equations with different time scales. Computing, 58, 173-185 (1997)

4. C. Gear, D. Wells, Multirate linear multistep methods. BIT, 24, 484-502 (1984)

5. M. Günther, A. Kværnø, P. Rentrop, Multirate partitioned Runge-Kutta methods. BIT, 41, 504-514 (2001)

6. E. Hairer, G. Wanner, Solving Ordinary Differential Equations II - Stiff and Differential-Algebraic Problems. Springer, Berlin (1996)

7. W. Hundsdorfer, V. Savcenco, Analysis of a multirate theta-method for stiff ODEs. To appear in Journal Appl. Num. Math.

8. W. Hundsdorfer, J.G. Verwer, Numerical Solution of Time-Dependent Advection-Diffusion-Reaction Equations. Springer, Berlin (2003)

9. J. Sand, S. Skelboe, Stability of backward Euler multirate methods and convergence of waveform relaxation. BIT, 32, 350-366 (1992)

10. V. Savcenco, Comparison of the asymptotic stability properties for two multirate strategies. Journal Comp. Appl. Math., 220, 508-524 (2008)

11. V. Savcenco, Construction of a multirate RODAS method for stiff ODEs. To appear in Journal Comp. Appl. Math.

12. V. Savcenco, W. Hundsdorfer, J.G. Verwer, A multirate time stepping strategy for stiff ordinary differential equations. BIT, 47, 137-155 (2007)

13. M. Striebel, M. Günther, A charge oriented mixed multirate method for a special class of index-1 network equations in chip design. Journal Appl. Numer. Math, 53, 489-507 (2005)

14. A. Verhoeven, A.El. Guennouni, E.J.W. ter Maten, R.M.M. Mattheij, A general compound multirate method for circuit simulation problems. In A.M. Anile, G. Ali, G. Mascali, editors, Scientific Computing in Electrical Engineering. Springer, Berlin Heidelberg (2006) 